\title{
Heart Rate Variability Characteristics Required for Simulation of Interval Sequences
}

\author{
FE Smith, EJ Bowers, P Langley, J Allen, A Murray \\ Medical Physics, Freeman Hospital, Newcastle upon Tyne, UK
}

\begin{abstract}
Fifty sequences of PhysioNet $R$-to- $R$ interval data, covering periods of between 20 and 24 hours, were classified into real or simulated groups. The RR interval characteristics were investigated in both the time domain and frequency domain. Eleven characteristics were analysed, and the range of measurements for each was studied for outliers from the main distribution. In the time domain, a restricted pattern of RR interval distributions classified 4 sequences as abnormal, and a reduced $R R$ variability produced 18 classifications, with an overlap of 8 , giving a total of 14/50 as abnormal. In the frequency domain, abnormally restricted very low frequency characteristics produced 26 classifications as abnormal with 10 overlaps giving a total of 16. The low frequency to high frequency ratio classified 4 as abnormal, but three of these were already detected by abnormal low frequency characteristics, giving a total of 17 classified in the frequency domain. Of the 17 classified in the frequency domain and of the 14 in the time domain there was an overlap of 9, resulting in 22 abnormal classifications, and suggesting that these were simulated. When PhysioNet assessed this classification a correct grouping of $100 \%$ was achieved on a single entry (reference 20020426.082234).
\end{abstract}

\section{Introduction}

The heart rate variability (HRV) signal non-invasively measures beat-to-beat alterations in heart rate, providing information about autonomic control of the cardiovascular system.

HRV is affected by both neural and hormonal inputs, at time intervals ranging from less than 1 second to 24 hours. Changes in HRV can relate to particular pathologies. Variability is known to decrease with age and among people who have medical conditions such as coronary heart disease, hypertension and diabetes mellitus. One of the main reasons for measuring HRV arises from its apparent ability to predict survival after myocardial infarction.[1,2] Despite current understanding of the mechanism of HRV the complex interdependencies of beat-to-beat variations at different time scales make it difficult to detect physiologically determined changes.

The aim of this study was to classify sequences of RR intervals as real or simulated by investigating the characteristics of heart rate variability, enabling simulators of beat-to-beat heart rate to be improved.

\section{Method}

\subsection{Data}

Fifty sequences of beat-to-beat intervals covering periods of between 20 and 24 hours were studied. They were made available from PhysioNet. Information provided by PhysioNet indicated that approximately half of the interval sequences were from real recordings of normal subjects and the remainder from automated simulators.

\subsection{Time domain analysis}

The 50 sequences of RR interval data were plotted, with inter-beat (RR) intervals on the $y$-axis and a time scale, in hours, on the $\mathrm{x}$-axis. Figure 1 shows examples of beat-to-beat RR interval sequences from the PhysioNet data.

The $50 \mathrm{RR}$ interval sequences were assessed in the time domain from six RR variability characteristics. $\mathrm{SD}_{\triangle \mathrm{RR}}$ (standard deviation of differences between adjacent $\mathrm{RR}$ intervals), $\mathrm{SD}_{\mathrm{RR}}$ (standard deviation of the averages of RR intervals in all 5-minute segments of the entire recording), $\mathrm{SD}_{\text {RRindex }}$ (mean of the standard deviations of all 5-minute segments of the entire recording) and $\mathrm{RR}_{50 \text { count }}$ (number of pairs of adjacent $\mathrm{RR}$ intervals differing by more than $50 \mathrm{~ms}$ in the entire recording).[3] Restricted patterns of RR interval distributions were investigated from the characteristics, $\mathrm{RR}_{\text {highcount }}$ (number of RR intervals $>0.8 \mathrm{~s}$ ) and $\mathrm{RR}_{\text {lowcount }}$ (number of RR intervals $<0.3 \mathrm{~s}$ ). 

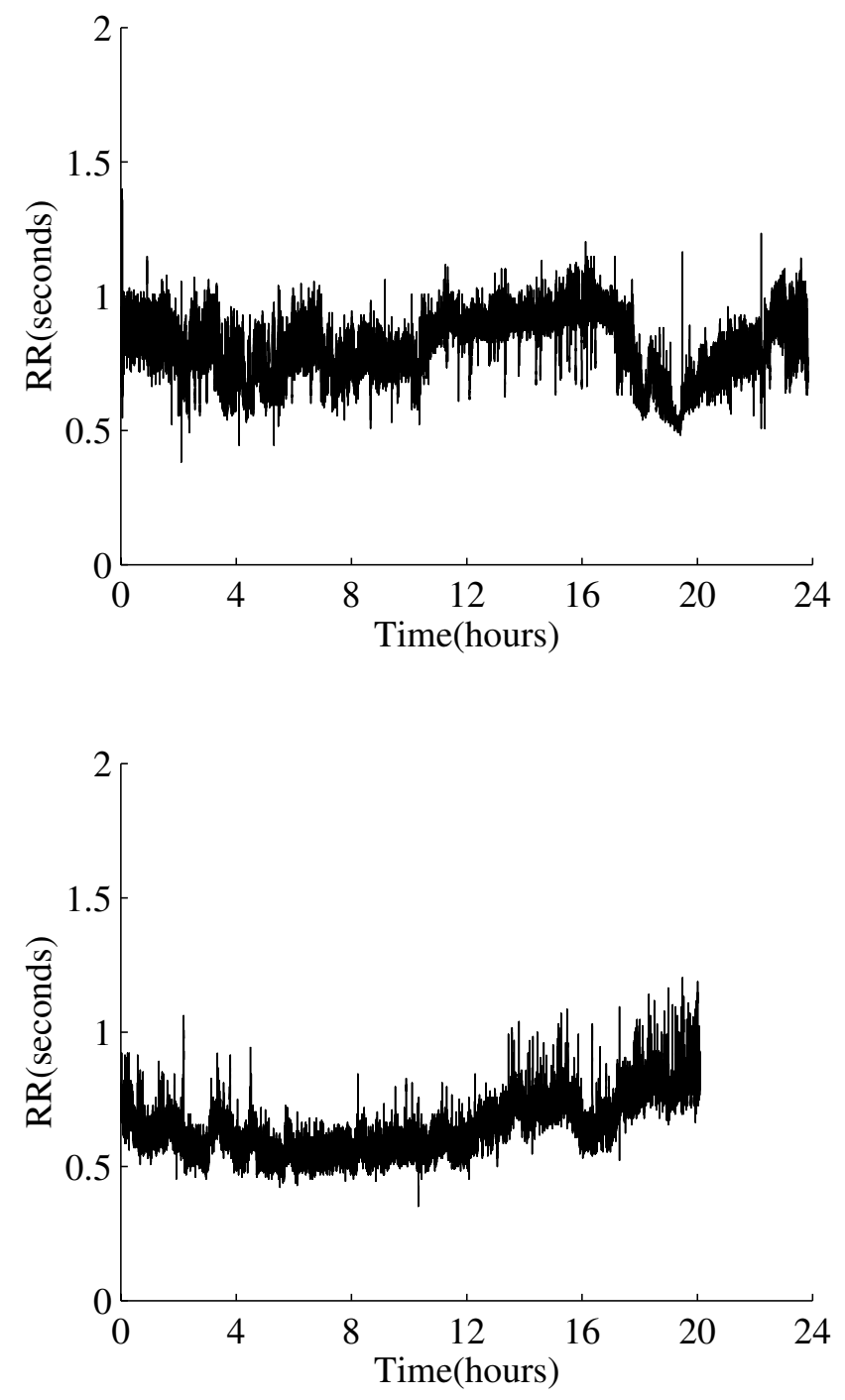

Figure 1 Examples of RR interval sequences provided by PhysioNet.

The range of measurements for each characteristic in the time-domain was studied for outliers from the main distribution. If the distribution showed no values that could be classed as outliers, the extreme 4 were classed as abnormal.

\subsection{Frequency domain analysis}

In the frequency domain a power spectral analysis was performed on the 50 sequences using a Fast Fourier Transform (FFT) in the beat domain.

Spectral analysis of the beat-to-beat data transformed the signal from time to frequency on the $\mathrm{x}$-axis, by representing the signal as a combination of sine and cosine waves, with different amplitudes and frequencies.
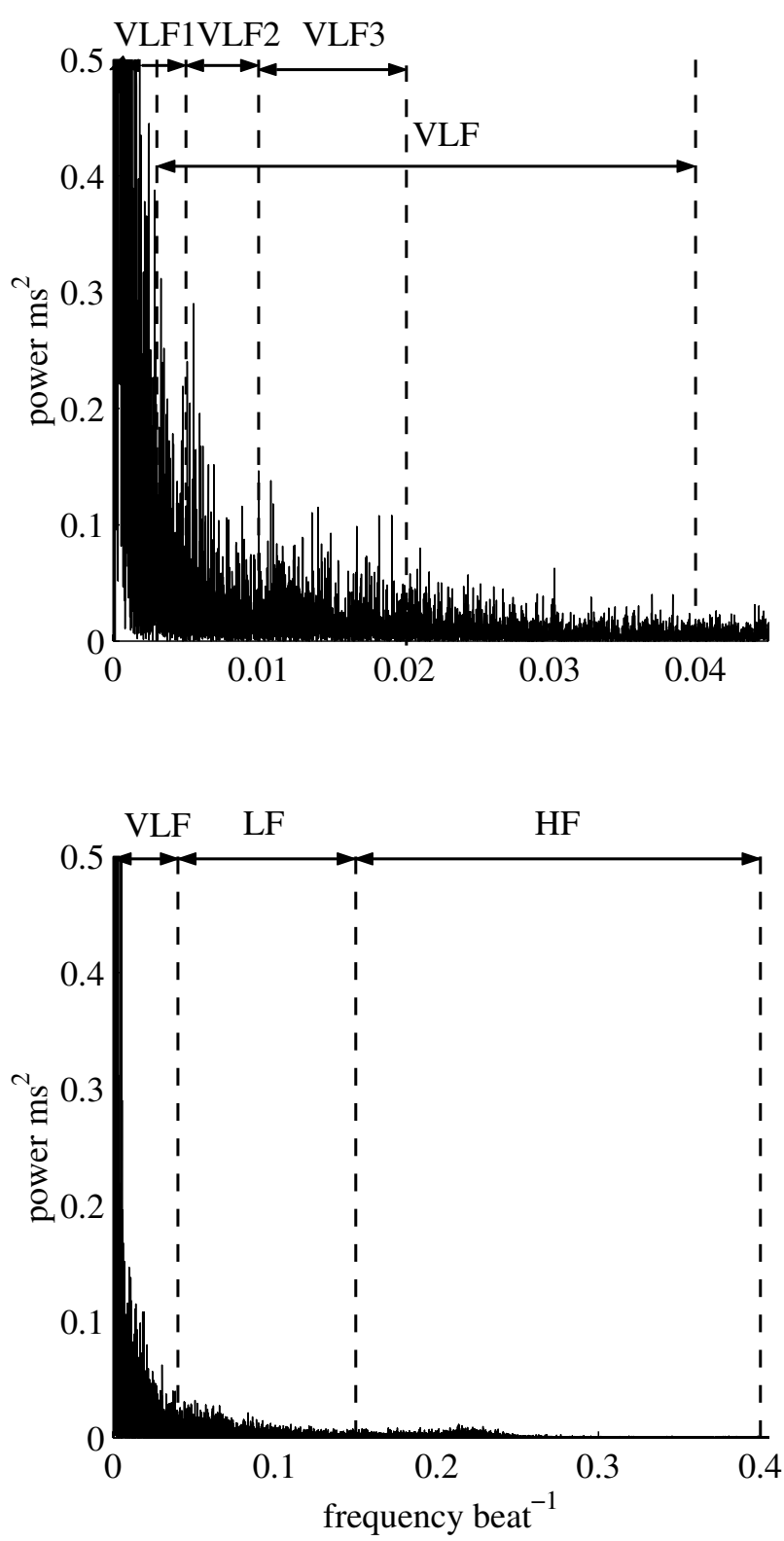

Figure 2 Example of a power spectrum for an RR sequence provided by PhysioNet.

Figure 2 shows an example of a generated power spectrum for one of the RR sequences provided by PhysioNet. The spectrum is divided into different frequency regions representing, very low frequency, VLF1 $(0.0001-0.005)$ beat $^{-1}$, VLF2 $(0.005-0.01)$ beat $^{-1}, \operatorname{VLF} 3(0.01-0.02)$ beat $^{-1}$ and VLF $(0.003-0.04)$ beat $^{-1}$, low frequency, LF $(0.04-0.15)$ beat $^{-1}$ and high frequency, HF $(0.15-0.4)$ beat $^{-1}$, shown on the diagram. The 50 PhysioNet RR data sequences were investigated in the frequency domain from the characteristic ratios, 
VLF1/VLF2, (VLF1+VLF2)/VLF3, VLF/LF, LF/HF and $\mathrm{VLF}_{\text {width }}$ (width of the power spectrum at half maximum).

The range of measurements for each characteristic in the frequency domain was studied for outliers from the main distribution using the same selection criteria as for the time domain analysis.

\section{Results}

Table 1 represents the classification results for the time domain and frequency domain analysis.

Table 1. Classification results for the time domain and frequency domain analysis.

\begin{tabular}{|c|c|c|c|}
\hline Domain & Characteristic & $\begin{array}{l}\text { Normal } \\
\text { range }\end{array}$ & $\begin{array}{l}\text { Number } \\
\text { of } \\
\text { abnormal } \\
\text { recordings }\end{array}$ \\
\hline \multirow[t]{6}{*}{ Time } & $\mathrm{SD}_{\Delta \mathrm{RR}}$ & $14-107 \mathrm{~ms}$ & 4 \\
\hline & $\mathrm{SD}_{\mathrm{RR}}$ & $72-231 \mathrm{~ms}$ & 6 \\
\hline & $\mathrm{SD}_{\text {RRindex }}$ & $31-111 \mathrm{~ms}$ & 4 \\
\hline & $\mathrm{RR}_{50 \text { count }}$ & $424-20484$ & 4 \\
\hline & $\mathrm{RR}_{\text {lowcount }}$ & $0-23$ & 2 \\
\hline & $\mathrm{RR}_{\text {highcount }}$ & $3968-127172$ & 2 \\
\hline \multirow[t]{5}{*}{ Frequency } & VLF1/VLF2 & $6-54$ & 6 \\
\hline & $\begin{array}{l}\text { (VLF1+VLF2)/ } \\
\text { VLF3 }\end{array}$ & $1-106$ & 4 \\
\hline & $\mathrm{VLF} / \mathrm{LF}$ & $0.1-5$ & 8 \\
\hline & $\mathrm{VLF}_{\text {width }}$ & $0.001-0.0025$ beat $^{-1} \&$ & 8 \\
\hline & $\mathrm{LF} / \mathrm{HF}$ & $0.2-1.5$ & 4 \\
\hline
\end{tabular}

In the time domain, a restricted pattern of $\mathrm{RR}$ interval distributions, $R R_{\text {highcount }}$ and $R_{R_{\text {lowcount }}}$ classified 4 sequences as abnormal, and a reduced RR variability, $\mathrm{SD}_{\Delta \mathrm{RR}}, \mathrm{SD}_{\mathrm{RR}}, \mathrm{SD}_{\mathrm{RR} \text { index }}$ and $\mathrm{RR}_{50 \text { count }}$ classified 18, with an overlap of 8 , giving a total of 14/50 as abnormal. In the frequency domain, the abnormally restricted very low frequency characteristics (VLF1/VLF2,

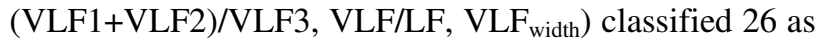

abnormal with 10 overlaps, giving a total of 16 . The low frequency to high frequency ratio $(\mathrm{LF} / \mathrm{HF})$ classified 4 as abnormal, but 3 these were detected by abnormal low frequency characteristics, giving a total of 17 classified in the frequency domain.
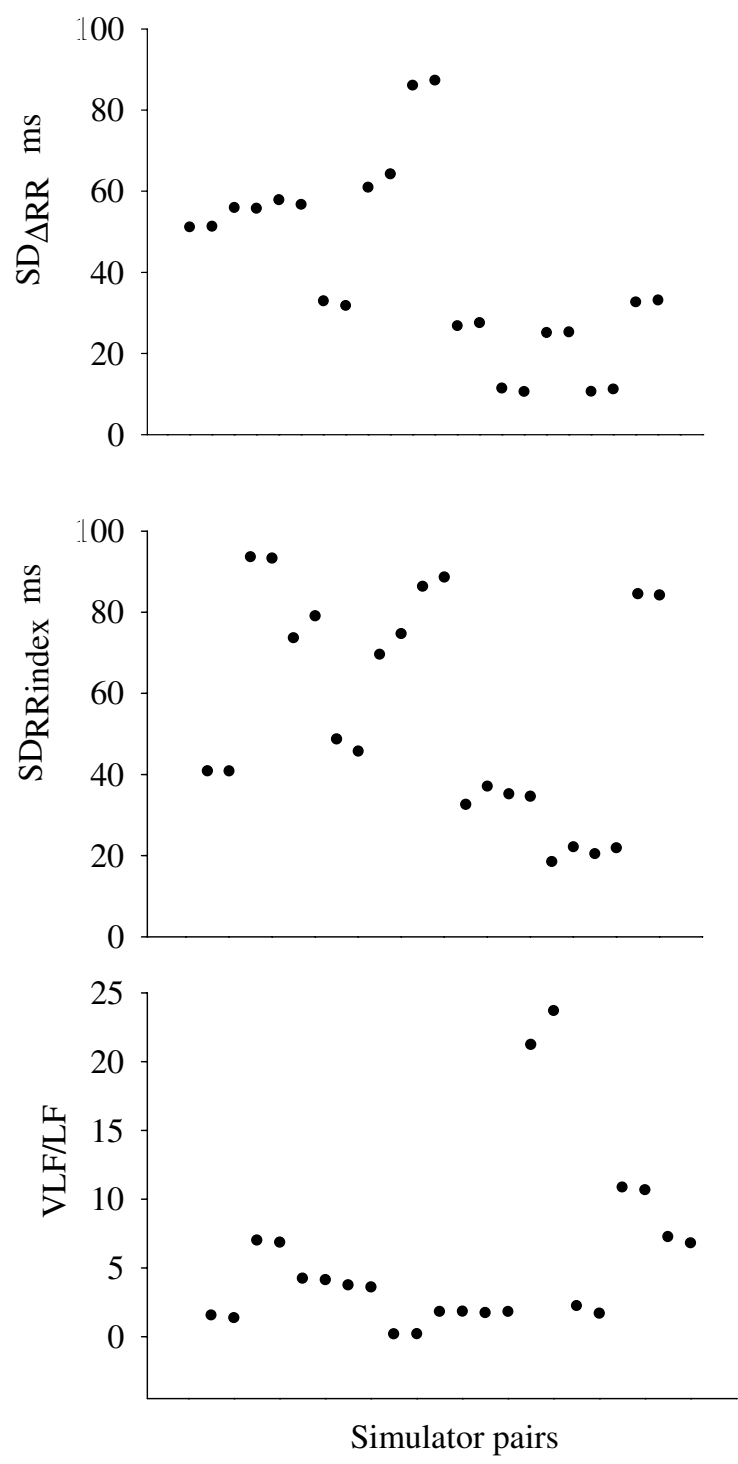

Figure 3 Examples of the pairs of values obtained from the simulated RR interval data for 3 of the characteristics investigated

Of the 14 classified in the time domain and the 17 classified in the frequency domain there was an overlap of 9 , resulting in 22 abnormal classifications, suggesting that these were simulated. When PhysioNet assessed this classification a correct grouping of $100 \%$ was achieved on a single entry (reference 20020426.082234). It was of note that the measurements for each of the characteristics 
assessed as abnormal tended to occur in pairs of strikingly similar values for the simulators, in agreement with the fact that two sequences for each simulator were included. Figure 3 shows examples of the pairs of values obtained from the simulated RR interval data for 3 of the characteristics investigated.

\section{Discussion and conclusions}

This study has used the characteristics of heart rate variability in order to classify $50 \mathrm{RR}$ sequences, from PhysioNet, as either real or simulated. We achieved a classification accuracy of $100 \%$, using time and frequency domain analysis to investigate unusual RR interval distribution and variability characteristics and skewed frequency distributions. Our investigation has shown that simulators need to have improvements in RR interval ranges, increased heart rate variability, and better very low frequency characteristics.

\section{Acknowledgements}

FES and PL are supported by the Engineering and Physical Sciences Research Council (EPSRC). EJB is supported by the special trustees of Newcastle upon Tyne Hospitals NHS Trust.

\section{References}

[1] Kleiger RE, Miller JP, Bigger JT, Moss AJ. Decreased heart rate variability and its association with increased mortality after acute myocardial infarction. AM J Cardiol 1987;59:256-62.

[2] Malik M, Farell T, Cripps T, Camm AJ. Heart-rate variability in relation to prognosis after myocardial infarction: selection of optimal processing techniques. Eur Heart J 1989;10:1060-74.

[3] Malik M, et al. Heart-rate variability: Standards of measurement, physiological interpretation and clinical use. Circulation 1996;93:1043-65.

Address for correspondence.

Fiona E Smith

Regional Medical Physics Department

Freeman Hospital

High Heaton

Newcastle upon Tyne

NE7 7DN

UK

f.e.smith@ncl.ac.uk 